\title{
ENHANCEMENT OF GALACTIC DYNAMOS BY DENSITY WAVES
}

\author{
Makoto TOSA and Masashi CHIBA \\ Astronomical Institute, Tohoku University, Sendai, 980 Japan
}

\begin{abstract}
We examine effects of density waves on the local galactic $\alpha \omega$-dynamo. Oscillations of the magnetic field and the dynamo parameters due to the density wave perturbation irreversibly couple with the dynamo action to enhance the growth of the magnetic fields.
\end{abstract}

\section{Introduction}

The standard galactic dynamo, the $\alpha \omega$-dynamo, works as a combined effect of the turbulence, the $\alpha$-action, and the shear of the differential rotation, the $\omega$-action ( see for reviews e.g. Parker 1979; Zeldovich et al. 1983; Ruzumaikin et al. 1988). If the spiral structure in the disk is taken into account, these actions are likely to be affected by the density wave perturbation to change the dynamo efficiency. In this paper we will examine the effect of the density wave on the galactic dynamo using a simple model and show that the density wave could enhance the efficiently of the galactic $\alpha \omega$-dynamo.

\section{Model Equations: A Simplified $\alpha \omega$-Dynamo}

The essence of the generation mechanism of magnetic fields by $\alpha \omega$-dynamo in the galactic disk can be seen in the local dynamo equation with thin disk approximation. Using dimensionless time and space variables normalized as $t \rightarrow t / h^{2} \eta$ and $z \rightarrow z / h$, where $h$ is the disk thickness and $\eta$ is the diffusion coefficient of fields, the local $\alpha \omega$-dynamo is described by the following equations (see e.g. Zeldovich et al. 1983; Ruzumaikin et al. 1988) :

$$
\frac{\partial B_{r}}{\partial t}=-R_{\alpha} \frac{\partial\left(\alpha_{1} B_{\theta}\right)}{\partial z}+\frac{\partial^{2} B_{r}}{\partial z^{2}}, \quad \frac{\partial B_{\theta}}{\partial t}=R_{\omega} B_{r}+\frac{\partial^{2} B_{\theta}}{\partial z^{2}}
$$

Here $R_{\alpha}$ and $R_{\omega}$ are dimensionless parameters characterizing the efficiencies of $\alpha$ - and $\omega$ - effects, and defined as $R_{\alpha}=\alpha_{0} h / \eta$ and $R_{\omega}=G h^{2} / \eta$, respectively; the $\alpha$-parameter characterizing the mean helicity of the turbulence is assumed as $\alpha(z)=\alpha_{0} \alpha_{1}(z)$, where $\alpha_{1}(z)$ represents the normalized z-distribution of the $\alpha$-parameter; $G$ is the shear parameter defined as $G=r(d \Omega / d r)$, and $\Omega$ is the angular velocity of the galactic rotation. 
These equations essentially describe the amplification of magnetic fields through the following chain of $\alpha$ - and $\omega$-actions: the radial field component $B_{r}$ is generated from the azimuthal component $B_{\theta}$ through $\alpha$-action arising from the helical turbulence as represented by the term $-R_{\alpha} \partial\left(\alpha_{1} B_{\theta}\right) / \partial z$; then $B_{\theta}$ is generated from $B_{r}$ through $\omega$-action arising from the shear of the galactic differential rotation as represented by the term $R_{\omega} B_{r}$. Repeating the chain of $\alpha$ - and $\omega$-actions, fields can be amplified.

To make the problem as simple as possible we restrict our consideration to the lowest even symmetric mode with respect to the galactic plane, which has simplest field configuration and the highest growth rate. Discarding the field structure in z-direction we consider the following simplified equations, instead of equations (1) and (2),

$$
\frac{d \tilde{B}_{r}}{d t}=\tilde{R}_{\alpha} \tilde{B}_{\theta}-\frac{\tilde{B}_{r}}{\tau_{d}}, \quad \frac{d \tilde{B}_{\theta}}{d t}=\tilde{R}_{\omega} \tilde{B}_{r}-\frac{\tilde{B}_{\theta}}{\tau_{d}} .
$$

The lowest even symmetric mode confined within a disk of thickness $h$ with no dynamo actions just decays with a time scale of $\sim 4 h^{2} / \pi^{2} \eta$, so that the dimensionless diffusion time is $\tau_{d} \approx 4 / \pi^{2}$ (see e.g. Zeldovich et al. 1983). These equations may be regarded as equations (1) averaged over the disk in $z$-direction, in which details of the averaging and the $z$-dependence of the physical quantities are absorbed effectively in the parameters $\tilde{R}_{\alpha}$ and $\tilde{R}_{\omega}$. Although these equations are too simple to derive detailed z-structure of the dynamo modes, they still correctly describe the chain of dynamo actions in the $\alpha \omega$-dynamo. The term $\tilde{R}_{\alpha} \tilde{B}_{\theta}$ represents the generation rate of $\tilde{B}_{r}$ by $\alpha$-action, and $\tilde{R}_{\omega} \tilde{B}_{r}$ represents the generation rate of $\tilde{B}_{\theta}$ by $\omega$-action. The terms $-\tilde{B}_{r} / \tau_{d}$ and $-\tilde{B}_{\theta} / \tau_{d}$ represent the loss of fields by diffusion in $z$-direction.

For the time evolution of fields as $\exp (\omega t)$, equations (2) yield $\omega=-\pi^{2} / 4 \pm|\tilde{D}|$, where $\tilde{D}$ is the dynamo number defined as $\tilde{D}=\tilde{R}_{\alpha} \tilde{R}_{\omega}$, and $\tau_{d}$ is assumed to be $4 / \pi^{2}$. This gives the critical dynamo number as $\left|\tilde{D}_{c 0}\right|=\left(\pi^{2} / 4\right)^{2}$; the dynamo instability occurs to increase the fields for $|\tilde{D}|$ larger than $\left|\tilde{D}_{c}\right|$.

For brevity we omit the tilde over the physical quantities in the followings.

\section{2. $\alpha \omega$ - Dynamo Modified by Density Waves}

Since the density waves strongly affect the gas and magnetic fields (Roberts and Yuan 1971; Tosa 1973), they can modify the dynamo action through the changes of the fields and the dynamo parameters. According to the standard density wave theory, in the spiral arm the interstellar gas is strongly compressed and star formation is induced, while in the inter-arm region the gas is decompressed and the star formation is suppressed( e.g. Roberts 1969). If the star formation excites the turbulence of the gas in the spiral arms, the $\alpha$-action will be enhanced in the spiral arms.

Another important consequence of the density wave perturbation is the change of the shear of the differential rotation. The shear parameter changes as the gas is compressed or decompressed by the density wave. In a disk with $\Omega(r) \propto r^{\gamma-1}$, if the gas is compressed by a factor $f$, i.e. $f=\rho / \bar{\rho}$, where $\bar{\rho}$ is the averaged density in the unperturbed disk, keeping the angular momentum unchanged, the shear parameter changes as

$$
G=\frac{(\gamma+1) f-2}{\gamma-1} \bar{G}
$$


where $\bar{G}$ represents the shear parameter in the unperturbed disk. Equation (3) indicates that the shear increases as the gas is decompressed, and vice versa.

Thus the density wave affects the $\alpha \omega$-dynamo in such a way that in the spiral arm the $\alpha$-action is enhanced but the $\omega$-action is reduced, while in the inter-arm regions the $\alpha$-action is reduced but the $\omega$-action is increased.

In order to illuminate the essential effect of the density wave, we simplify the density wave in the following way. As the fields are convected with the gas in galactic rotation, they are subjected to the density wave oscillation. Following the galactic rotation we divide a period of the density wave oscillation into two parts, compressed phase in the spiral arm and the decompressed phase in the inter-arm and assume that in each period the dynamo parameters are constant.

To simplify the problem further, we assume that $B_{r}$ is essentially generated in the spiral arm through $\alpha$-action, while $B_{\theta}$ is generated in the inter-arm regions through $\omega$-action; thus in the spiral arm the dynamo parameters are $R_{\alpha}=f_{\alpha} \bar{R}_{\alpha}, R_{\omega}=0$, and $\rho=\rho_{s}=f \bar{\rho}$, where the bar indicates unperturbed quantities in the disk without density waves and $f, f_{\alpha}$, and $f_{\omega}$ are assumed to be constant; in the inter-arm, $R_{\alpha}=0, R_{\omega}=f_{\omega} \bar{R}_{\omega}$, and $\rho=\rho_{i}=\bar{\rho} / f$. With these assumptions and simplifications, equations (4) and (5) can be easily integrated.

At the interface between the inter-arm and arm, we impose the following boundary conditions. Since the compression or decompression of the gas by the density wave is essentially in radial direction, $B_{r}$ is continuous across the boundary between arm and interarm regions, and only $B_{\theta}$ changes as the gas density changes. We assume that $B_{\theta}$ changes or jumps across the boundary in proportion to the variation of the density. Thus at the interface from the inter-arm to the arm or from the arm to the inter-arm, the densities are related as $\rho_{i}=f^{-2} \rho_{s}$, and hence $B_{\theta s}$ in the arm is related to that in the inter-arm $B_{\theta i}$ as $B_{\theta s}=f^{2} B_{\theta i}$.

Starting from a given value of the fields and the phase of density wave oscillation, the simplified dynamo equations (3) are integrated and the solutions in the spiral arm and the inter-arm are connected by the relevant jump conditions. If we compare the field strength after one cycle of the density wave oscillation, the increase of the field strength can be calculated; the ratio of the final field strength to the initial one gives the amplification factor of the field generation. If the field strength is greater than that at the beginning of the cycle, the field grows, and vice versa.

\section{Periodic Solution and the Critical Dynamo Number}

The steady state, which provides a critical condition for the field amplification, is obtained by the periodic condition for the field variation where, after one cycle of the density wave oscillation, the field returns to the value at the beginning of the cycle. The periodic condition yields the critical dynamo number as:

$$
\left|\bar{D}_{c}\right|=\left|\left(\bar{R}_{\alpha} \bar{R}_{\omega}\right)_{c}\right|=\frac{\left(1-\exp \left(-\tau / \tau_{d}\right)\right)^{2}}{f^{2} f_{\alpha} f_{\omega} t_{i} t_{s}} e^{\tau / \tau_{d}},
$$

where $\tau_{s}$ and $\tau_{i}$ are durations of the spiral and inter-arm phases, respectively; $\tau$ is the period of one density wave oscillation and $\tau=\tau_{s}+\tau_{i}$. The critical dynamo number $\bar{D}_{c}$ is defined by the parameters in the unperturbed disk, and provides a critical state of the filed 
amplification in the presence of the density waves. Equation (4) indicates that the critical dynamo number is reduced by the factor $1 /\left(f^{2} f_{\alpha} f_{\omega}\right)$ by the presence of the density wave perturbation.

The increase of the shear in the inter-arm due to the decompression of gas can be calculated from equation (3). For a flat rotation curve $(\gamma=0)$, decompression of gas by a factor $1 / 2$, i.e. $f=2$, for example, increases the shear by a factor $3 / 2$ and hence increases the $\omega$-parameter by the same factor. The increase of the $\alpha$-action in the spiral arm is expected but is difficult to estimate quantitatively because our present knowledge on the generation of turbulence in the spiral arm is too poor to allow us to make quantitative estimation of $\alpha$-action or $f_{\alpha}$. Thus we just assume that $f_{\alpha}>1$.

To compare the critical dynamo number with that of a uniform disk,$D_{c 0}$, derived from the simplified dynamo equations (2), consider a limit of $\tau \rightarrow 0$. If we take $f_{\alpha}=1, f_{\omega}=1$, and $\tau_{a}=\tau_{i}=\tau / 2$, the present dynamo just generates fields through alternative actions of $\alpha$ - and $\omega$ - effects without density wave perturbation. In the limit of $\tau \rightarrow 0$, the critical dynamo number becomes $D_{c} \rightarrow 4 / \tau_{d}^{2}$, which is 4 times that of a dynamo described by equations (2). This factor 4 comes form the fact that in the present model the $\alpha$ - and $\omega$ actions work alternatively; while one action is working the other one is sleeping. Taking account of the factor 4 we have

$$
\frac{\bar{D}_{c}}{D_{c 0}}=\frac{4}{f^{2} f_{\alpha} f_{\omega}}
$$

Thus even moderate variation of gas density and dynamo parameters due to density waves significantly reduce the critical dynamo number; hence magnetic fields can be amplified with the help of density wave even in a disk which has lower dynamo number than the critical value required for the field amplification in the absence of the density wave.

The present work was supported in part by the Scientific Research Fund of the Ministry of Education, Science and Culture (61540173,63540184, and 01540210)

\section{References}

Parker,E.N. 1980, Cosmical Magnetic Fields(Oxford: Clearendon Press).

Roberts,W.W. 1969, Ap.J.,158,123.

Roberts.W.W., and Yuan,C., 1971, Ap.J,161, 877.

Ruzumaikin,A.A, Shukurov,A.M., and Sokoloff,D.D. 1988, Magnetic Fields of Galaxies, (Dordrecht: Kluwer Academic Publishers).

Tosa,M., 1973, Publ.Astron.Soc.Japan,25,191.

Zeldovich,Ya.B.,Ruzumaikin,A.A.,and Sokoloff,D.D.1983, Magnetic Fields in Astrophysics, (New York: Gordon and Breach). 